\title{
Annexe: Voltaire dans la presse francophone éditée en Russie sous Elisabeth
}

\section{I : Voltaire dans le Caméléon littéraire, St.-Pétersbourg, 1755}

[5 janvier 1755, t. 1, nº 1, p. 3-5]

DE ST PETERSBOURG. Il paroit ici depuis quatre jours une nouvelle réponse de $\mathrm{Mr}$. DE LA BEAUMELLE à Mr. DE VOLTAIRE, c'est toujours le même objet, toujours les remarques critiques du premier sur le Siècle de Louis XIV du second : ce procès ridicule est porté depuis long-tems au tribunal du public, mais je pense que ces Messieurs se sont réservés le droit d'apeller du jugement : Rodrigue qui l'eut-dit? chimène qui l'eut cru ? que le chantre de Henri, seroit un jour vilipendé par l'auteur du Qu'en dira t'on ? Je laisse volontiers guéroïer nos Champions, ou plutôt Mr. DE LA BEAUMELLE tout seul, puisque son adverse ne daigne pas seulement [p.4] lui rendre injure pour injure ; mais au moins qu'il ait la bonté de ne pas faire passer pour disputes littéraire, une mauvaise chicanne qui n'intérèsse nullement les savans, \& qui n'est autre chose qu'une maussade joutte de personalités \& d'invectives, soutenue d'un stile vif \& petillant fort de mots mais vuide de chose, je rend justice, Mr. DE LA BEAUMELLE écrit avec un feu que voudrois avoir, c'est une tournure un Phrasisme sésuisant, mais est on auteur à ce prix ? possèder les graces de sa langue, y joindre d'usage du monde, \& du gout pour le leger voilà ce qui donne le stile, de $\mathrm{M}$ pour les avoir fait passéra t’il donc pour auteur? d'ailleurs au cas particulier c'est le four qui apelle le moulin brulé, que l'on me pardonne le proverbe ; un échapé de la bastille reproche à son antagoniste qu'il est chassé de tous les païs : ah ! Mr. DE LA BEAUMELE dire qu'il ne reste à VOLTAIRE que COLMAR \& votre pitié, y pensés vous ? n'y à t'il donc plus de....\& ne vaudroit il pas mieux cesser de vivre que d'exciter en vous ce sentiment dont votre orguëil se pare : il vous sied mal sur le ton de censeur de passer en revüe les ouvrages d'AROÜET ; je le disois avant-hier vous étes à Mr. DE VOLTAIRE ce qu'est un point géométrique au nombre 1000, car enfin, un petit retour sur vous-même. [p. 5] Croïés cependant que je ne m'erige point en DON QUICHOTTE des réputations attaquées, je n'aime point à combattre des moulins à vent, vous m'entendés. Ainsi Messrs. à votre aise, dites vous réciproquement vos vérités, j’y consens : mais ; du coté des mœurs, du caractère, de la conduite, des sentimens. Nous verons de jolies choses, \& je vous livre l'auteur de ZAÏRE : prenés garde à vous cependant, il est homme à prendre sa revanche, tâtés vous bien : quant à l'esprit, au savoir, au mérite, au talent, pavillon bas : ou si vous étés assés vain pour vous croire au pair d'un grand homme, \& en même tems assés teméraire pour le mépriser, faites usage du conseil que vous lui donés vousmême « le mépris est froid, \& vous l'étes si peu : soïés le donc».

[12 janvier 1755, t. 1, n², p. 38-44]

EXTRAIT. L'on mande de Paris que Monsieur de VOLTAIRE \& Mr de CRÉBILLON vont dans peu, l'un \& l'autre donner au Théâtre Français une Tragédie nouvelle dont le sujet est le même, sous des noms différens. Monsieur de CRÉBILLON intitule sa pièce 
Annexe: « Voltaire dans la presse francophone »

le Triumvirat, VOLTAIRE nomme la sienne la mort de CICÉRON. Le public attend avec impatience ces ouvrages; frapés tous deux de main de maître ils peuvent être également beaux chacun dans leur espèce.

Rien n'est si avantagueux pour la littérature que ces luttes d'esprit \& de talens, surtout quand les atlettes sont à peu près de force égale. L'imagination de l'homme est presque infinie \& peut varier de mille façons sur un même objet. Ainsi trente auteurs [p. 39] tous fameux, s'il est possible d'en suposer un aussi grand nombre, peuvent traîter parfaitement une même matière chacun dans leur genre quoique par différentes métodes. L'axiôme il n'est qu'une façon de faire du bon me semble trop général. Cette règle a ses exceptions : j'en citerai volontiers un exemple qui me conduira à l'examen d'une pièce de Monsieur de MARMONTEL. Elle peut avoir encore les graces de la nouveauté au moins pour le pays étranger puisqu'elle n’a été jouée à Paris qu'en Juin 1752. \& imprimée en 1753. Je serais charmé que ce petit extrait pût inspirer le desir de la lire, \& que la satisfaction des conaisseurs justifiât le plaisir qu'elle m'a fait. C'est mon Tic, je n'aime point à ètre bien aise tout seul.

Les HÉRACLIDES, tel est le titre de cette pièce. Le sujet n'en est pas neuf. Une famille persécutée qui cherche azile, un [p. 40] Oracle qui ordonne le sacrifice d'une fille illustre sans s'expliquer, un peuple menacé de périr si l'Oracle ne s'accomplit, voilà ses ressemblances avec Iphigénie, \& l'on s'étonne que la même intrigue puisse produire un nouvel intérêt. DÉJANIRE veuve d'HERCULE persécutée par EURISTHÉE tyran d'ARGOS se retire avec ses enfans \& IOLAS ami d'HERCULE chés DÉMOPHON fils de Thésée \& Roi d'Athènes: au moment que Démophon fatigué d'une longue guerre contre Euristée est prêt à conclûre la paix. Le tyran aïant découvert la retraite de DÉJANIRE met pour condition au Traité qu'elle lui sera rendue ; mais STÉNÉLUS fils de DÉMOPHON séduit par les charmes d'OLIMPIE fille de DÉJANIRE engage son père à protéger ces infortunés. IOLAS découvre la fourberie de COPRÉE envoyé d'ARGOS il en rend compte à Sténélus, la guerre se ralûme mais avant de livrer bataille, DÉMOPHON consulte les Dieux. [p. 41] La réponse obscure de l'Oracle menace la perte de la bataille à moins que l'on n'immole une fille de sang illustre. DÉMOPHON incertain en confère avec IOLAS. OLIMPIE l'aprend, explique l'Oracle pour elle-même, \& se dévoue courageusement à la mort. Sa mère au désespoir accuse DÉMOPHON d'artifice \& veut mourir pour sauver sa fille : ce combat de la nature et du sentiment fournit les plus beaux traits.

Cependant OLIMPIE court au temple, déjà le coûteau du sacrifice est levé. STÉNÉLUS dans son camp découvre par un esclave ARGIEN, que le Grand-Prêtre gagné par COPRÉE a fait parler l'Oracle ; il vole à l'autel, arrache sa maîtresse à la mort, livre bataille, défait EURISTHÉE, remet le fils de DÉJANIRE sur le Trône d'ARGOS \& reçoit enfin le prix de son courage par une union que l'amour d'OLIMPIE, la reconnaissance [p. 42] de DÉJANINE \& la justice de DÉMOPHON aprouvent.

Tel est le précis de cette pièce dont tous les rôles sont également beaux, sans que l'un brille aux dépens de l'autre. Le plus parfait \& le plus rare, à mon avis, est celui de DÉJANIRE que je mets sans hésiter audessus de toutes les Reines que j'ai vû jusques ici au théâtre. Les vers sont nobles et aisés, la pièce est pleine de ces sentences qui saisissent \& que le spectateur se plaît à retenir. En voici quelques traits. Act. I Sc. 2.

Le peuple est à son prince, \& son Prince est à lui ;

Et de leurs intérêts l'accord ferme et paisible

Rend le Roi tout puissant \& le peuple invincible : 
La pensée de Monsieur de VOLTAIRE, que vous faut il de plus, MÉROPE est à vos pieds est doublée bien délicatement par DĖJANIRE au pied de DÉMOPHON. Act I. Sc. 4 [p. 43]

Par mon abaissement jugés de ma tendresse

Jugés, surtout, jugés du péril qui les presse.

Un Français en parlant de l'opiniatreté du Clergé de l'église Gallicane, rebelle aux ordres de son Roi pourait en dire ce que dit DÉMOPHON Act. II Sc. 8 à propos des pontifes du Temple.

Ils ont placé le trône au pied de leurs autels

Et les Rois devant eux ne sont que des mortels.

Que ces vers sont beaux dans la bouche de DÉJANIRE au sujet de la supertition ! Act. IV Sc. 3 .

Déchirés le bandeau que les homes perfides

Étendent sur les yeux des peuples trop timides ;

Démasqués, confondés, leur pieuse fureur,

Il reste un hydre à vaincre, \& cet hydre est l'erreur :

Osés la terasser, cette seule victoire

D’Hercule \& de Thésée effacera la gloire.

[p. 44] Et ces autres Act. V

Ô Superstition ! ô fille des Enfers !

De quels monstres affreux tu remplis l'univers !

Tu fais de l'homme un Tigre \& ta noire imposture

Dans le cœur le plus tendre étouffe la nature.

[9 février 1755, t. 1, n6, p. 128-131]

\section{NOUVELLES DE PARIS}

L'on vient de voir à la Cour de Fontainebleau un spéctacle d'un genre nouveaux; c'est Daphnis \& Alcimadure, pastoralle languedocienne, précédée d'un prologue Français qui a pour titre Isaure ou les jeux Floraux. Les paroles, \& la musique de la pastoralle sont du Sr. MONDONVILLE, mâitre de musique de la Chapelle du Roi, \& les ballets de Sr. LAVAL, mâitre des ballets de Sa Majesté. On à aussi éxécuté un nouveau ballet Héroïque intitulé Anacréon. Les paroles sont de S. de CAHUZAC, la musique du SR. RAMEAU \& la danse de Sr. LAVAL. Les talents supérieurs du Sr. GELIOTTE faisant appréhender sa retraite du théatre, qui était fixée à pâques prochain ; Sa Majesté pour l'engager à rester, vient de lui accorder une pension de six mil livres sa vie durante, \& en outre une somme de trente mil livres d'argent comptant pour païer ses dettes. Les Srs. PIRRON, de CAHUZAC \& MARMONTEL viennent aussi d'obtenir du Roi, chacun une pension de deux mil livres.

Le Sr. PREVILLE qui avait été admis à la comédie française pour jouër les troisiémes [p. 129] amoureux, vient de recevoir l'ordre de jouër les valets après le Sr. ARMAND \& le Sr. DUBOIS. Le théatre de Paris est un de ceux ou l'on récompense le mieux les talents, mais aussi un de ceux ou l'on fait moins de quartier aux sujets qui manquent 
de merite. Le Sr. BARON qui jouoit les petits confidents dans le Tragique, \& seconds rôles de parsans ou bouche trous dans le Comique vient d'être renvoïé sans miséricorde, sans même lui laisser achever son année. Bel exemple \& que l'on devrait suivre dans nombre de troupes, un Directeur trop complaisant sur pareil chapitre en est la duppe, \& le public en souffre, le théatre est fait pour amuser en instruisant.

Si chacun n'y restait que le temps de son rôle

Tout serait à sa place \& l'on ne verait pas

Tant de gens inutils dont le Public est las.

\section{GRESSET}

On à enfin donné le Triumvirat, Tragédie nouvelle du Grand Crébillon attendüe avec impatience de la part des amateurs du COTHURNE, mais la montagne est encore acouchée d'une Souris; L'on pourrait presque dire de cette piéce ce que le Poëte LINIĖRE $^{1}$ à dit [p. 13o] du Poëme de la Pucelle par CHAPELAIN, voici son Epigramme.

Nous attendons de Chapelain,

Ce rare \& fameux Ecrivain,

Une merveilleuse Pucelle.

La cabale en dit force bien ;

Depuis vingt ans on parle d'elle,

Dans six mois on n'en dira rien.

Le fort du Triumvirat à été fâcheux dés le premier jour, quoiqu'il y ait de très beaux vers répandus au hazard ; mais selon l'usage moderne le dernier acte est pitoiable, la pièce est froide et point du tout intéréssante. Il n'y a qu'une seule femme, dont le rôle voudrait être beau, mais en tout il est manqué, quoique Mademoiselle CLAIRON le rende dans la derniére perféction. ANTOINE qui devrait avoir une si grande part dans cette fameuse révolution ne paraît que derriére la toile, \& CICERON qui selon toutes les aparançes n'est pas dans les bonnes graçes de CREBILLON est aussi petit garçon dans ce poême que dans son CATILINA. Ce sera aujourd'hui la septiéme réprésentation, \& l'on craint que ce soit la derniére, ou du moins celà frisera bien la chute. Les Comédiens qui s'attendent à ces [p. 131] événement ont répeté VENCESLAS, qu'ils vont remettre au Théatre assitôt après le retour de Fontainebleau. Ils ont donné Nanine Comédie de Mr. de V. qui dans sa nouveauté eut un très médiocre succès, mais à cette reprise elle en a eû un prodigieux. On va repéter incéssament une Tragédie nouvelle de la composition de l'Auteur des TROÏENNES, qui a six pièces toutes prêtes. Voilâ bien de la besogne que nous avons devant les mains.

[16 février 1755, t. $1, \mathrm{n}^{\circ}$ 7, p. 143-147]

\section{REFLEXIONS D'UNE FEMME D'ESPRIT}

La véritable Philosophie n'est pas celle qui se perd en raisonemens vains \& subtils, mais celle qui conduit droit à la morale, qui enseigne le chemin de la vertu, \& qui finit par remettre l'homme entre les bras de la Religion. Cette dernière est la science du bonheur, l'unique sourçe de la joïe, de la tranquilité, l'unique trésor dans toutes les situations de la vie, l'unique asile dans les afflictions, l'unique consolation dans la mort. Ce sont les salutaires principes qui nous inspirent l'amour de la vertu, \& qui nous la

\footnotetext{
${ }^{1}$ François Payot de Linière (1628-1704), auteur de chansons libertines et de couplets satiriques.
} 
font regarder non comme un fardeau gênant, mais comme la plus agréable [p. 144] compagne que nous puissions avoir dans notre voyage à travers le monde.

Il serait à souhaiter que tous les hommes soient raisonables \& que tous les hommes raisonables soient heureux.

Les flatteurs sont mil fois plus nuisibles à la vérité \& aux bonnes moëurs que les esprits fort \& toute la sequelle des libertins.

Il vaut mieux rendre service à un homme d'un état abjet qu'a des persones d'une condition plus relevée : parce qu'il est à craindre qu'un grand ou un riche ne veuïlle païer que nous lui rendons, \& ne nous mette par là dans une dependance, qui nous fasse ésclave de ses passions ou ministre de ses volontés.

Il est impossible à un homme dans le malheur de calmer son esprit, soit par le voïe de la réflexion, soit en se livrant uniquement à l'impression des sens ; si ceux-ci nous égarent, celle-là nous tourmente. Nous paraissons acquiésçer à la dispensation d'en haut ; mais nous nous éfforçons à l'ajuster toujours [p. 145] avec le plan de notre cöeur. C'est ce plan qui est le centre ou nous raportons tout : on voudrait le voir acompli, on voudrait que ce fût bientôt, l'on est désolé des circonstançes qui s'y oposent. Ce revers porte le trouble au-dedans de nous, le cöeur veut en quelque sorte s'en vanger sur l'esprit ; il y répand des nuages, \& tâche d'offusquer la lumière importune.

Extrait de la C. S.

Les vérités essentielles ne peuvent être remises trop souvent sous les yeux du lecteur, \& j’ai crû que des réfléxions préséntées par ce séxe enchanteur, dont l'apanage principal semble être le don de persuader, feraient une plus vive impression ; d'ailleurs j'en prens moi-même ocasion de raisoner ; \& j'imagine que ce n'est point une chose à négliger.

Le livre ou j'ai puisé ces maximes est un Roman allemand. Que l'Heroine de l'Auteur soit un être chimérique on réel, qu'il lui prête des sentimens, des pensées, ou qu'en éffet ce soit les siennes cet éxamen m'importe peu. D'une ou d'autre façon, je suis tou[p. 146]jours bien fondé à répondre aux Sots de ma nation, qui pretendent que l'esprit, l'imagination, le jugement, sont anexés à leur seul païs, \& qui poussent la fatuité jusqu'à croire, quil faut être français pour penser, pour écrire, pour créer quelque chose de bon ou d'utile. Abstraction faite des arts qui marchent chés bien d'autres nations d'un pas égal au progrés qu'ils font chés la mienne : je soutiens que les talens de l'esprit de presque tous les peuples policés de l'Europe ne le cédent en rien à mon païs. Le stupide préjugé de Patriotisme ne m'aveugle point, j'admire le beau par tout ou je le trouve ; \& je suis choqué des defauts que j'aperçois dans les Auteurs français, peutêtre même plus que je ne le ferais de la part d'un autre. Le mérite particulier d'un Ecrivain ne m'en impose point sur ses torts ; \& le fameux Chantre de HENRI sera toujours à mes yeux un Philosophe sans principes, un Historien de mauvaise foi ; ce terme n'est pas trop fort ; il est absurde à un quelqu'un qui détaïle des faits historiques de s'excuser, en disant qu'il a peutêtre été mal informé ; lorsque l'on n'est pas sûr de ce que l'on avançe on doit prendre le parti du silençe, singulièrement lors-[p. 147]qu'il est question d'un grand homme. Le nom de PIERRE I. est trop réspectable \& trop auguste pour oser rien hazarder, qui ne soit conforme au vrai, l'Auteur de Zaïre me saura gré, j'espere de ne 
Annexe: « Voltaire dans la presse francophone »

pas lui citer plus précisement sa faute à cet égard. C'est un affront que je lui épargne aux yeux de tout l'Univers; il me permettra seulement de lui rappeler qu'il n'était permis qu'au seul Apelle de peindre le portrait d'Alexandre.

[23 février 1755, t. 1, n8, p. 167-176]

APOLOGIE DE L'ORTHOGRAPHE DE MONSIEUR DE V...

Certain auteur à défini l'Ecriture. un art ingénieux

De peindre la parole \& de parler aux yeux,

Et par des traits divers, \& figures tracées,

Donner de la couleur \& de l'ame aux pensées.

BREBEUF

Je trouve cette définition complete, elle exprime en peu de mots les qualités propres \& les avantages immenses que la société civile retire de cet art que l'on nomme jûstement ingénieux. Je laisse aux Historiens le so[p. 168]in de remonter à son origine \& de nous expliquer si nous en sommes redevables aux Egiptiens, aux Caldéens, ou aux Phéniciens.

Le Poëte LUCAIN l'attribüe à ces derniers :

Phenices primi, famae si creditur, aussi

Mansuram rudibus vocem signare figuris.

Le Sr. BILLETTE DE FANIERE qui a l'exemple de BREBEUF s'est ingéré de traduire LUCAIN nous a rendu les deux vers latins par les quatre suivans.

C'est des Phéniciens que nous vient l'art d'ecrire,

Cet art ingénieux de parler sans rien dire,

Et par des traits divers, que notre main conduit,

D’attacher au papier la parole qui fuit.

J'ai dit ailleurs que les traductions dégradent toujours l'original. Passons la dessus.

Il est constant que l'écriture est un moïen de doner du corps aux pensées. Il est donc essentiel que ce corps soit tellement propre à [p. 169] peindre l'objet que l'on veut représenter, qu'il ne soit pas possible de s'y méprendre. Un mot de doit pas être susceptible d'une double signification qui peut induire à quantité d'erreurs. Mon but en raisonant sur cette matière, est de me justifier aux yeux de quelques personnes éclairées dont je réspecte infiniment les avis, \& qui m'ont fait un petit reproche de m'être écarté du sentier battu par l'usage pour suivre l'Ortografe de Mr. de V., l'on aura la bonté de m'excuser, si je traite cet article superficiellement ; c'est pour éviter une Dissertation purement Grammaticale qui sent toujours le Pédantisme. Je me borne donc à présenter quelques considérations.

Monsieur de V..... est d'un mérite distingué et réconu : l'admiration que j’ai pour le grand homme n'est pas cependant le motif qui me décide en faveur de son Ortografe. 
On peut être excellent Poëte, bon Historien, Philosophe habile, sans conaître à fond les règles de l'Ecriture, cet article est du res[p. 170]sort du Grammairien, Monsieur de V... ne serait pas moins un grand homme quand il l'ignorerait. Ce n'est donc pas l'esprit de parti n'y la prévention qui me guide ; l'Evidence seule a droit de me convaincre ; elle me paraît sensible à l'égard de la nouvelle métode dont j'enreprens la défense. Il y manque dit-on le sceau de l'aprobation Académique, d'abord ; mais question de savoir, si faute de cette ratification la métode est mauvaise en elle-même. J'ose soutenir le contraire. On est surpris qu'un seul homme fasse tête à un corps de savans, mais, on ne doit pas l'être que cet homme trouve des imitateurs, lorsqu'il sait persuader par des raisonemens solides.

Tout est de convention dans la vie ; c'est à la convention que l'on doit la diversité des langues, celle des mots emploïés pour la signification des choses, celle des Caractères propres à former les mots, \& à peindre la pensée par le cours de l'Ecriture. Mais les hommes en [p. 171] formant cette convention, n'ont pas juré de s'y tenir inviolablement sans jamais y ajouter ou soustraire. Par conséquent le sic voluere Patres n'a plus lieu, \& c'est prouver bien faiblement la valeur d'une pratique, d'un usage, que d'alleguer la volonté anciene \& primitive de ceux qui l'ont mis en vogue.

Rien n'est parfait, rien ne peut jamais le devenir. La perfection est un attribut de l'essence divine, \& ne saurait avoir lieu pour les choses humaines ; mais on peut si non y attendre du moins en aprocher. L'on ne doit négliger aucun soin à cet égard, c'est une émulation digne d'éloge, nécéssaire au bon ordre, utile au bien de la société. Suivons ce raisonement dans chacune de ses parties.

S'il est difficile d'arriver à la perfection, il est constant qu'une chose dans son principe en est beaucoup plus éloignée, qu'après une suite de tems, des progrés soutenus, des observations, des variations, des rafinemens. Jusqu'a présent voilà Mr. de V.. autorisé. [p. 172] Il n'a point interverti l'ordre, il n'a point empiété sur la respectable vétusté. Mais par zêle il a réfléchi, ses réfléxions lui ont découvert des abus, il essaie de les réformer; je ne trouve dans toute cette conduite qu'un savant qui ambitionne d'être utile à sa Patrie \& au public. L'est il éfféctivement. Cette proposition est aussi facile à démontrer que la précédente.

I. Tout ce qui peut contribuer au Laconisme touche à l'élégance, celle-ci est mere de la persuasion, voici déjà un grand gain pour le stile. Nous en avons l'obligation à Monsieur de V., il a frappé nombre de superfluïtés, qui ne servaient qu'a rendre le Phrasisme prolixe, \& à répandre un louche désagreable, sur des pensées qui voudraient être vives et pétillantes.

II. Rien n'est plus avantageux que de fixer les incertitudes de l'Etranger, \& celles même de la nation en prenant le parti d'écrire les mots tels qu'ils doivent [p. 173] être lus ; c'est sauver un ridicule à la Langue Française. Il est douteux encore si l'on doit écrire comme l'on parle, ou prononcer comme l'on écrit, \& l'on ne fait éxactement ni l'un ni l'autre graçes à l'usage qui prévaut impunément. J'ai conu un axiôme en droit qui revient ici fort à propos. Abusus non est usus, un abus ne fait pas loi, au contraire on doit savoir gré à ceux qui s'en rendent réformateurs.

Il y a eu de tous tems de ces génies solides, assés habiles pour sentir l'absurdité, \& assés francs pour crier à l'erreur : sans cela nous parlerions encore Gaulois. Je lis dans les Cent nouvelles, dans Bocace, dans les nuits de Straparo'e, gaigner pour gagner, grant pour grand, ors daleine, pour hors d'haleine, aultre pour autre: d'où vient cette 
réforme? on répond que la langue s'est épurée, que l'on a retranché certaines lettres qui formaient à l'oreille un son choquant, \& ajouté quelques autres qui ont parû nécéssaires [p. 174] pour donner plus de force à l'expression. En ce cas on a donc porté atteinte à cet usage dont on réclame aujourd'hui le déspotisme contre Monsieur de V.... qu'a-t-il fait de plus que ces premiers restaurateurs ? il a trouvé mauvais p. e. qu'un nom d'homme s'écrivit comme celui d'un peuple, tandis que tous deux ont un son différent. François ne doit pas se confondre avec Français ; c'est un qui pro quo, \& puisque l'on est d'acord dans notre langue que oi sonera différament que $a i$, pourquoi s'ecarter de cette rêgle générale? pourquoi y mettre ces excéptions chimériques qui n'ont d'autres base que la corruption de l'usage ? de foire à faire la différence est totale : en suivant strictement les principes que Monsieur de V... critique, foire \& faire doivent se prononcer également, si français peut s'écrire avec un oi. Je prens ce seul mot comme un des plus ordinaires \& je m'y retranche, un plus grand detail me conduirait à des volumes.

[p. 175] Tel est en bref le résumé du sistême de Monsieur de V... \& les raisons qui me déterminent d'y souscrire. Les lettres ne sont faites que pour être prononcées, n'en emploïons donc jamais d'inutiles, \& s'il le faut ajoutons en de nécéssaires. Evitons surtout l'équivoque \& l'amphibologie, aplanissons à l'étranger un chemin facile qui le conduise sans degout à la connaissance de notre langue dont les principes arbitraires \& contradictoires le revoltent. Si l'Anglais a sur nous l'avantage de penser solidement, aïons au moins, celui de dégager notre Idiôme d'un fatras d'inutilités, \& d'écrire enfin comme nous parlons. L'Académie à refusé d'adopter cette métode, c'est domage pour le public que ce corps respéctable ne soit pas d'acord sur un article aussi éssentiel, mais on ne perd au plus que le tems d'attendre, cet évenement n'est peut être même pas loin. Nous avons vû des choses condamnées pendant plusieurs années \& réçues ensuite : témoin le miroir ardent d'Archimède avec lequel il embrasa la flotte des [p. 176] Romains, suivant que l'Histoire raporte. Ce fait a été tenu pour apocrif \& fabuleux, parce que l'on ne conaissait encore que la forçe des miroirs Sphériques, dont l'effet est beaucoup inférieur. Depuis quelques années Mr. de BUFFON a trouvé la composition d'un miroir de plusieurs pièces qui multiplie l'action des raïons solaires \& qui brûle à deux cents pas, fond le plomb à cent, \& l'argent à cinquante. On en conclût que le miroir d'Archimède étoit peutêtre de la même espece \& l'on fait réparation de l'Histoire. Le sistême de Monsieur de BUFFON est recû, il est vrai qu'il est un des quarante, mais Monsieur de V... n'y mérite t'il pas une place?

[9 mars 1755, t. 1, n 10, p. 222-229]

\section{EXTRAIT.}

J'ai lû à la tête de la Sémiramis de Monsieur de VOLTAIRE une dissertation sur le Théatre ancien \& moderne, dans laquelle on reconaît le stile de l'auteur même de la pièçe. Peu de persones sont à portée d'avoir vû cette Dissertation; elle mérite néanmoins une attention particuliere, parcequ'elle donne une idée exacte des progrés du Théatre depuis les Grécs jusqu'a notre Siécle, on y trouve d'ailleurs, une comparaison de tous les Théatres, \& une apréciation d'eux, j'ai crû faire plaisir de la présenter en abrégé sous les yeux du lécteur. 
Sous le Pontificat de LEON X, le Théatre dit l'auteur renâquit ainsi que l'eloquence : c'est dans ce tems que le Prélat TRISSINO mit au jour sa Tragédie de Sophonishe, premiére piéçe que l'Europe ait vüe ; [p. 223] la Calandra du Cardinal BIBIENA peu auparavant avait été la premiére Comédie dans l'Italie moderne.

Depuis les beaux jours d'Atênes, la scêne tragique languissait, plusieurs héritiers du talent des Sophocles \& des Euripides lui ont enfin rendu ses premiers honneurs. L'Opera Italien est de tous les spéctacles celui qui nous donne une image plus vraie de la Scêne grécque ; le récitatif Italien ressemble à la Mélopée des anciens. Le gout du chant pour l'arlette, n'a néanmoins nul raport au chant gréc, un héros meurt aujourd'hui comme les Cignes : à Atênes ÖEDIPE \& ORESTE auraient parus trèsridicules s'ils eussent au moment de la reconaissance frédonés de petits airs \& débité des comparaisons à Elêctre \& à Jocaste.

L’Opera Français est encore beaucoup plus éloigné du Théatre gréc; nôtre déclamation est trop languissante dans la Mélopée, [p. 224] \& trop précipitée dans l'ariette ; nous tombons dans le Dialogue \& dans toutes les petites maximes coupées qui ne produisent que des chansons, fort aprochantes à celles des BARCAROLES de VENISE. Thésée par exemple sur le point d'être empoisoné par son Père lui chante cette fadeur

Le plus sage

S'enflamme et s'engage

Sans savoir coment.

Morale d'Opera, ce nom est passé en proverbe en l'emploiant on anonce une frivolité. Le Théatre Italien jouit d'un avantage bien supérieur. Les maximes qui s'y débitent sont d'une forçe majéstueuse, \& le célébre Métastase s'est rendu par les Operas rival des CORNEILLES \& des RACINES. Qu'on en juge par cette magnifique sentence (éternelle leçon pour les Rois] \& qu'il a scu plaçer habilement dans la bouche de Titus. [p. 225]

..... il torre altrui la vita

E facolta commune

Al piu vil della Terra ; il darla è folo

De numi, \& de regnanti

Le plus vil des hommes a le pouvoir d'oter

la vie à son semblable; mais il n'appartient

Qu'aux Dieux E aux Souverains de la donner.

Sentimens dignes des Sophocles pourquoi faut-il que vous soïés si rares de nos jours ? pourquoi faut-il que notre Scéne n'ait conservé de vous que le préstige des chants de la danse, de la décoration \& des machines; faible succés! qui n'est dû qu'a un enchantement superficiel.

La bonne \& la vraïe Tragedie répare heureusement la perte du Théatre Gréc, surtout en France. Mairet a l'imitation de la Sophonishe de TRISSINO, à le premiér introduit la régle de l'unité de temps, de lieu \& d'action. Nos tragédies en suprimant le [p. 226] coturne, le masque \& les déclamations outrées se sont rapprochées de la simple nature. L'Histoire a pris la plaçe de la fable, \& si la politique, l'ambition, la jalousie, les fureurs de l'amour regnent sur nos Théatres, ce n'est que pour en faire conaître le danger par des éxemples frapans. La Tragédie est l'ecole de la vertu ; le Théatre épuré \& les livres de morale n'ont qu'une légère différence. Sur la Scêne l'instruction est réduite en action; elle en devient plus intéréssante. D’ailleurs les mêmes pensées qui seraient 
Annexe: « Voltaire dans la presse francophone »

infiniment plus faibles dans la prose, deviennent plus sublimes \& plus vives relevées des graçes \& des charmes de la Poësie ; art ingénieux d'instruire la Terre \& de loüer le ciel, \& que par cette raison l'on appelle, le langage des Dieux.

Les inventeurs ont toujours la premiére place dans la mémoire des hommes, \& quoique notre Siécle singuliérement ait poussé le Tragique à un degré de perféction qui surpasse de beaucoup les Grecs, on ne peut re [p. 227] à ces premiérs la gloire d'être les peres de Coturne. Le mérite d'Homere n'est point attenué par les beautés du Tasse. Euripide n'est soit éclipsé par l'auteur de BAJAZET, mais peutêtre s'ils eussent été contemporains le Poëte grec aurait il apris avec plaisir cet art de préparer finement le spectateur à tous les événemens qui vont passer sous ses yeux ; celui du choc des passions, du combat dé sentimens, \& de ces situations ménagées \& interéssantes ; art négligé par les inventeurs même de l'art. Au reste les Grecs \& les modernes ont un grand nombre de parités. Parler dignement \& avec liberté, peindre les mœurs \& les réformer, traiter des sujets de pure imagination, \& en tirer les leçons les plus utiles. LE PERE BRUMOI dans son Théatre des Grecs observe assés mal à propos que la Tragédie ne peut souffrir de sujets feints \& que jamais on ne prit cette liberté à Atênes ; «La raison dit-il c'est que l'esprit humain ne peut être touché que de la vraisemblançe. » Pitoiable raisonement comme si [p. 228] dans un sujet d'invention le vraisemblable ne pouvait pas être gardé ; dans Zaïre tout est controuvé jusqu'aux noms. La vraisemblance y est elle choquée ? d'ailleurs il est faux que les grecs n'aient point eû cette espéce de Tragédie. ARISTOTE dit positivement qu'Agaton s'est rendu célébre dans ce genre. Il est constant en outre que ces sortes de sujets réussissent; ils sont même plus difficiles que d'autres, \& c'est-là que le génie \& le Talent du Poëte se develope le mieux. Le seul reproche que l'on puisse faire légitimement à ces sortes de piéçes, c'est qu'ordinairement elles sont un Tissu d'intrigues amoureuses. Despreaux dit en vain.

...... de l'amour la sensible peinture

Est pour aller au cœur la route la plus sûre.

La route de la nature est plus certaine \& plus noble : les morceaux les plus frapans d'Iphigénie sont ceux ou Clitemnestre défend sa fille, non pas ceux ou Achille défend son [p. 229] Amante. Le cœur n'est qu'efleuré par des complaintes amoureuses, il est véritablement êmu, pénétré de la situation douloureuse d'une mere d'un père, d'un fils, plus on retranchera l'amour du Théatre plus il fera l'ecole epurée des mœurs \& de la vertu.

[16 mars 1755, t. 1, n¹1, p. 232-243]

Dissertation sur l'origine et les progrès de la poésie; fondée sur le témoignage des anciens auteurs grecs et latins, auquel on a joint celui des auteurs modernes. Traduction d'un manuscrit en langue Russe

[p. 236] [...] il me siérait mal de vouloir fixer les principes d'une science que je ne pratique point. Mais je veux traiter de l'etendüe des conaissances qu'exige l'art de la Poësie, des peines inséparables des qualités éssentielles qui constituent un véritable Poëte, de ce don particulier qu'il doit avoir recû du ciel, ce Talent de penser, de s'élever 
au-dessus de lui-même par la saillie d'une imagination vive, cette Tournure d'expressions sublimes, en un mot ce tout que l'on ne sait pas exactement définir, mais que l'on apelle avec Justice feu Poëtique : (*) présent de la nature, qu'elle seule peut nous faire, qui ne s'acquiert ni par l'étude ni par aucun effort; present qu'elle accorde à peu de persones, quoiqu'un grand nombre se flatte d'en joür, présent sans lequel Rousseau n'eut été qu'un rimailleur; [p. 237] E à raison duquel l'on ne conaît au monde qu'un ROUSSEAU, un CORNEILLE, un RACINE, un GRESSET, un VOLTAIRE.

(*) Ou plutôt entousiasme. Les Sibilles à ce que disent les anciens, étaient agitées d'un feu secret au moment de la prophétisation, un Poëte animé, rempli de son sujet ressemble assés aux Sibilles.

[23 mars 1755, t. $1, \mathrm{n}^{\circ} 12$, p. $\left.268-274\right]$

\section{PENSÉES}

La vertu se fait aimer \& rèspècter dans les disgraces, c'est-là quelle s'epure, quelle se perfectione, quelle jouït de tout son éclat ; la pompe et la fortune marchent rarement à sa suite : l'orgueil \& la présomption accompagnent presque toujours ceux qui sont dans le bonheur. La douceur au contraire \& la modestie qui sont l'apanage des infortunés rendent sensibles pour eux. Un charme secret attendrit en leur faveur. Avant. de Calliope par Monsieur L.B.

Deux cœurs en bonne intèlligence, vivent sous l'empire de l'amour, tendres sans jalousie, fideles sans contrainte, heureux sans indiscrétion.

[p. 269] Le père de NEUVILLE dans l'oraison Funebre du Cardinal de FLEURI, enseigne avec bien de l'éloquence ce que c'est que servir l'etat « c'est dit-il, décrier, confondre, proscrire à jamais dans l'ésprit d'une nation, la basse \& rampante ambition qui marche aux honneurs par des voies dont rougit la vertu; l'indolente ou présomptueuse ambition qui se repose sur les honeurs sans zêle ou sans capacité pour en soutenir le poids ; la coupable \& funeste ambition qui se sert des honneurs que pour se livrer avec impunité à la licence des passions. »

Les hommes à qui la nature a donné beaucoup d'ésprit et d'aptitude pour les sciences, n'ont qu'à la feconder en se livrant à elle avec confiance ; c'est lorsque la nature a peu donné qu'il faut presque la violenter pour supléer à ce qu'elle a refusé.

[p. 270] Rien n'est si précieux que le tems, rien cependant dont on fasse moins de cas. Senéque dit que le tems est presque toujours partagé en trois: Que l'on en passe une partie à ne rien faire, une autre à mal faire, \& la dernière à ne pas faire ce qu'on doit. Monsieur de VOLTAIRE a donné un discours assés fort dans ses pièces de litteratures sur ce que l'on ne fait pas, \& sur ce qu'on devrait faire.

Les Princes, les Rois, les personnes, constituées en dignités \& utiles à l'état doivent faire un meilleur emploi du tems que les autres hommes. VESPASIEN disait « il faut qu'un Prince meure debout, Suetone vie de VESPASIEN, pour exprimer qu'il ne doit jamais être sans faire quelque-chose d'utile à sa patrie. 
L'Empereur Julien à -dit qu'un Philosophe \& un galant-homme ne doivent pas seu[p. 271] lement respirer. Il entendait par -là que c'est perdre le temps de donner aux besoins du corps, au-delà de ce que l'on ne peut leur refuser.

Muley Moluch Roi de Fez dans la bataille qu'il gagna en 1578 sur SEBASTIEN Roi du Portugal. Ce générexu more acablé de vieillesse ne voulut jamais abandonner son armée ; absorbé par la fatigue qui épuisa les restes de sa vie, il expira le doigt sur la bouche, comme pour recomander à ses sûjets de ne pas perdre le tems à le regretter, mais qu'ils l'employassent à battre l'ennemi.

Je me rapelle une chanson qui contient d'agréables maximes elle a pour Titre $* * *$

[p. 272]

L'USAGE DU TEMS

$* * *$

Tout ici bas prendra fin,

Tout fuit la triste destinée,

Celui qui naît le matin,

N'est pas sûr de l'après-dinée :

Content des ordres du destin

En bûvant de ce jus divin,

Je cherche à jouïr du présent

Qui seul peut faire un sort charmant.

$* * *$

[p. 273]

***

Donner au passé des fleurs,

Trouvés-vous que c'est etre sage?

Réflêchir sur les malheurs,

En revient-il quelque avantage?

L'on met le plaisir dans l'oubli ;

Et le bien le plus accompli ;

Je crois donc la réfléxion

Sur les chagrins hors de Saison.

$* * *$

Qui veut prévoir l'avenir,

Se trouble \& brouille sa cervelle,

Je me livre à mon plaisir,

Quand il s'enfuit je le rappelle :

[p. 274]

Dès que je sens qu'il veut passer, Je cherche à le recomencer ;

Ainsi je jouis du present,

Et mon cœur est toujours content. 
[6 avril 1755, t. $2, \mathrm{n}^{\circ} 14$, p. 303-318]

\section{LETTRE DE L'AUTEUR A SON AMI.}

Vous avés grande raison mon cher, si j'avais conu tout l'embaras inséparable d'une feuille périodique, je ne me serais pas chargé d'une comission si délicate. L'amour du travail, un peu d'ambition \& le desir de tenir un coin dans le monde ont été mes motifs. La réflexion vient trop tard lorsqu'elle tombe sur des objets dont elle n'a pas su prévoir les conséquences. J'ai suivi mon goût sans consulter mes forces ; j'espérais qu'on me tiendrait compte des efforts, le public trop rigoureux pour faire grace à la bonne intention juge sans [p. 304] ménagement \& l'auteur \& l'ouvrage ; je tâche en vain de le satisfaire. Chaque jour me découvre de nouvelles difficultés. Celle de créer du neuf d'est pas sans doute la moindre, surtout lorsqu'elle se reproduit d'une semaine l'autre. Tout est tellement épuisé qu'il reste bien peu de choses à dire ; il faut un génie supérieur pour découvrir des omissions après tant d'habiles Ecrivains. J'ai cru sauver cet inconvénient en variant les sujets, \& m'étendant peu sur le même j'avais compté me réserver des ressources. Cette méthode ne plaît point, on voudrait en seize pages trouver un ouvrage fini ; \& lorsque par hazard je raisonne un peu plus que de coutume sur une seule matière cette uniformité fait naître l'ennui. Conseillés-moi de grâce, ou j'abondone tout. Le Caméléon change de couleur, à l'abri de ce titre frivole je me croyais la diversité permise ; préjugé de ma nation, que le Nord n'adopte point ; je pourais amuser en France par cette cascade qui ne fatigue pas l'attention, ici l'on veut être fixé. Je [p. 305] vais perdre tous mes avantages si l'on m'ôte celui de la legèreté.

C'est sans doute faire l'éloge d'une nation que de vous la montrer plus raisonable que la nôtre, mais dites-moi mon cher ! ai-je tort, il me semble que c'est imoler le plaisir à un goût décidé pour le sérieux, il me paraît même injuste d'exiger qu'une bagatelle qui ne doit occuper qu'un moment de lecture attache aussi solidement qu'un ouvrage réfléchi. Peut-être avec le tems ferai-je goûter mon Sistême; sans être opiniatre je ne m'en départirai qu'à toute extrémité. Au reste gardés-vous de m'énvoyer jamais de nouveautés, quoique l'on en soit assés avide dans ces climats ; j'ai cru faire merveille en les publiant au moins par extrait, ce caractère de vérité qui ne me quittera jamais, m’a fait parler avec trop de franchise \& l'on m'acuse de passion.

Bon Dieu! que ne gardiés-vous cette mort de CICÉRON, dont pour mon malheur [p. 306] j'ai pensé trop haut, on m'acuse de VOLTAIROMANIE, m'avés-vous jamais conu sur ce ton. Touché du vraîment beau, choqué du faux \& du fade, j’admire l'un, \& fuis sans quartier pour l'autre. Dans une Tragédie je sépare les vers de la pièce, j'aplaudis au langage des Muses, \& trouve la pièce en elle-même mauvaise, est-ce ma faute après tout, si la conduite est manquée, si l'intrigue n'a pas un certain ordre, si les Scênes sont cousues avec du gros fil, si les héros ne sont pas de grand-homes, si un sentiment contredit l'autre, si le dénoûment ne ressemble à rien. Tout mon tort est d'avoir un peu manqué à la loi du respect qu'un jeune-homme doit à la vieillesse. Eh bien ! dorenavant je dirai que Catilina vaut mieux que Rome sauvée, que Rhadamiste est au dessus de Zaïre : mais on ne me croira pas, que faire ? je ne dirai rien : il est trop dangereux de parler. Je m'en souviens pour avoir dit dans une Dissertation sur la 
Poësie (Ouvrage qui fait honeur au pays, \& à l'auteur) [p. 307] pour avoir dit faire des vers c'est peu de chose, en faire de bons, c'est quelque chose, ne faire que cela ce n'est rien j'ai pensé... mais après tout j'éntendais parler d'une nation en général, je disais que c'est trop borner le goût, le talent \& le mérite d'un pays de le réduire simplement à la Poësie, surtout quand l'expérience journelle démontre que les autres sciences, les arts y fleurissent avec un égal succès, je prétendais (car dans ce Siècle il faut développer toutes ses pensées) je prétendais que ce serait doner une trop legère Idée de la Russie, par Exemple, ne la louer qu'en partie, de publier les noms des Racines qu'elle produit, de ceux qui cherchent à s'y égaler. J'ajoutois, que, faire des vers, c'est peu de chose pour un Empire qui en fourni d'aussi grandes en tout genre ; en faire de bons, continuais-je, c'est déjà quelque chose, mais ne faire uniquement que cela, ce n'est rien. Non, rien en comparaison d'une infinité de productions utiles \& savantes qui rendent cette nation rivale du [p. 308] surplus de l'Europe, sans parler d'une florissante Académie formée sous les auspices du plus grand des Monarques (a) sans dire mot d'une Université naissante, \& qui s'élève sous le règne glorieux de l'auguste \& digne Fille d'un Héros (b). Les Russes n'ont-ils donc pas d'ailleurs cent endroits recomandables \& faits pour être admirés. Loix sages, gouvernement habile \& prudent, oeconomie judicieuse, armées triomphantes \& formidables, discipline militaire, police exacte, magistrature éclairée, Sénat auguste, Sénateurs dignes de l'ancienne Rome, un d'entre eux surtout plus utile à la patrie que dans le Cabinet, que les Scipions à la tête des légions, également propre néanmoins à les comander. Et dans le détail des caractères particuliers, bienfesance[,] urbanité, franchise, j'avais raison sans contredit, faire des vers c'est peu de chose, au moins les Muses devraient-elles s'exercer sur ces sujets, bien dignes d'être chantés.

(a) PIERRE Premier

(b) ELISABETH PETROWNA

[p. 309] Voilà, mon cher Ami, les griefs que l'on a contre moi, je ne puis y répondre c'est ce qui me désole. Et par quelle raison dirés-vous ; parcequ'il me sied mal de luter contre des persones que je respecte \& de l'avis desquelles je voudrais toujours être. Tenés, par exemple, votre fatal Triumvirat me met dans le cas de contredire un des Esprits les plus respectables, les plus polis \& les plus cultivés. je vous ai là de belles obligations ; vous avés fait de jolies choses, je parîrais que votre Abbé de St. PIERRE que vous m'ordonés absolument de lire me mettra encore aux prises avec quelqu'un. Cependant je le lirai, je l'ai déjà fait en partie ; j'admirerai sans doute avec vous ; me conviendrait-il de ne pas estimer un auteur si généralement considéré. Mais voudriésvous aussi qu'entraîné par le torrent je devinse un fade adulateur, \& que je donnasse tête baissée dans les travers les moins palliables. Non, mon cher, au risque même de votre [p. 310] indignation, je ne quitterai point la sage habitude de l'impartialité.

C'est-ce me semble un défaut que l'on ne peut reprocher à l'Abbé de St. PIERRE on le taxerait plus justement de donner dans l'oposé. Qu'il jouïsse, j’y consens, de la réputation d'un Politique habile, qu'il marche d'un pas égal sur cet article avec les ROUSSETTES \& les PUFFENDORFF, le vœu général que j'ai fait de ne jamais raisoner sur les affaires d'état, m’a toujours éloigné de l'étude qui conduit à la Politique, je m’y conois mal ou point du tout \& n'ai rien à dire à cet égard. Mais je ne digère point la façon libre \& presque hardie dont l'auteur s'explique sur le compte des deux plus 
grands Monarques de notre Siècle (c). De deux choses l'une, ou l'auteur ne nous donne que des conjectures ou ses remarques sont vraies. Au premier cas je trouve absurde de hazarder des raisonemens à

(c) PIERRE Premier \& Charles XII.

[p. 311] moins d'être bien informé, lorsqu'il s'agit de deux grands-hommes : au second je trouve au moins aussi déplacé de raconter des choses désavantageuses à la mémoire de deux personages illustres, de faire des réflexions sur leur conduite, \& de doner l'essor à sa Critique sur des objets dignes de la plus respectueuse admiration. Quand je lis un Ouvrage je m'assurettis peu à l'ordre des volumes à moins que la matière ne l'exige essentiellement; le IX. Tôme de l'Abbé de St. P. m'est tombé sous la main, j'y trouve Réflexions sur la vie de PIERRE Premier \& sur celle de Charles XII. de quel droit un Politique veut-il relever les fautes imaginaires de ces deux Souverains. Qu'il laisse à l'Histoire le soin de publier leurs vertus sans cacher leurs défauts, c'est elle qui toujours vraie doit ne rien omettre de ce qui les concerne. Mais ce rôle ne va point au politique Abbé. Ses distinctions me choquent, un grand-homme \& un grand-homme sont à l'entendre deux êtres différens, ce titre à mon sens ne peut se di[p. 312]viser \& n'est aplicable qu'à un Héros vaste dans ses entreprises, prudent dans ses démarches, ami de son peuple, père de ses sujets \& qui ne néglige rien pour leur donner des témoignages utiles et authentiques de son amour. Quelles sont ces différentes parties de la bienfesance dont l'Empereur \& le Roi de Suéde ne conaissaient pas l'usage ? ce premier sur tout a-t'il rien négligé de ce qui constitue cette vertu si recomandable dans l'esprit de l'auteur? sciences, arts, talens, établissemens, marine, art militaire, commerce, manufactures, lui est-il rien échapé. Voilà le grand-homme \& non pas celui qui comme dit l'Abbé de St. P. n'a d'autre vue que de gagner le Paradis. Pieux motif \& bien louable mais qui ferait plutôt des faints que des Conquérans. Lorsque l'auteur distingue les talents des actions, il pense juste, mais il s'explique mal, je crois, en disant qu'un homme peut-être grand du côté des actions sans l'être du côté des talents, \& reciproquement. Un Artiste du côté des talens n'est simplement [p. 313] qu'un habile homme, terme qui n'est point du tout le sinonime de grand. Mais un Héros, grandhomme par conséquent, joint les talens aux actions.

Au reste, mon cher ami, je ne m'étendrai point sur cet objet.

A vaincre sans péril on triomphe sans gloire.

Malheureusement, Monsieur de St. PIERRE n'est plus à portée de réfuter mes faibles objections. On croirait à coup sûr que je n'attaque que parceque je nai rien à craindre. D’ailleurs je ne vous donne ici que ma façon de penser particulière et me garde bien d'en cautioner la justesse. Je vous avouerai néanmoins que s'il est aussi vétilleux Moraliste que Politique scrupuleux, c'est peut-être ma faute si les beautés de son ouvrage me flattent moins que vous, je le regrette.

Parlons d'autre chose ; que dit-on chés vous des affaires du Clergé de France [...]

[4 mai 1755, t. 2, nº 17, p. 388-391]

LETTRE EN VERS AU MARQUIS DE PESÉE PAR SA MAITRESSE. 
Annexe: «Voltaire dans la presse francophone »

On la trouva dans sa cassette après qu'il fut tué dans une Bataille en Italie

Voudras tu, chèr amant, parmi le bruit des armes

Entendre le récit de mes vives allarmes?

Et quand Mars dans ton sein allume ses fureurs,

Tes yeux daigneraient ils voir une Amante en pleurs?

Quel trouble, quel éfroi, de mon âme s'empare!

Il court un bruit confus, qu'un combat se prépare,

Que vainement $C$... cherche à se retrancher,

Bruit cruel, chaque mot m'épouvante \& me glace ;

Pressentirais-je hélas ! une affreuse disgrace.

Ah ! je fais que la gloire a pour toi trop d'apas,

Que l'honeur au péril précipite tes pas.

Pour un Guerrier, tes yeux ont pour moi trop de charmes,

Pour un Amant, ton cœur aime trop les allarmes.

[p. 389]

Le ciel devait dumoins te rendre en te formant,

Ou moins vaillant guerrier, ou moins aimable Amant.

De mon sexe timide ignorant la faiblesse,

Je suis faites au péril ainsi qu'à la tendresse.

Que ne m'est il permis de voler après toi !

Si je suivais tes pas je n'aurais plus d'éfroi.

J'irais braver la mort, \& serais toujours prête

A m'exposer aux coups qui menacent ta tête.

Ta jeunesse, tes traits, tes cheveux, tes apas,

Ton teint vif, qu'Apollon ne désavourait pas,

Dans l'empire amoureux inévitables charmes,

Pour toi dans les combats sont d'inutiles armes ;

(Un homicide plomb avec impunité

(Frape sans respecter l'âge ni la beauté

Adonis

(*) C'est être assurement bien hardi de comparer des ouvrages de Monsieur de Voltaire, sa supériorité est si bien établie, que c'est risquer beaucoup de vouloir lui trouver des défauts. Aussi n'est ce point par un de ses vers, mais seulement pour détromper ceux qui m’acusent d'une prévention assés forte pour encenser jusques aux torts de ce grand-homme, suposé qu'il en eût dans quelques uns de ses ouvrages. Je crois avoir remarqué par

[p. 390]

Adonis autrefois, comme toi fut aimable ;

Pour toi je crains, hélas ! son destin déplorable :

Vénus entre ses bras lui vit perdre le jour ;

Je n'ai point ses attraits, mais j'en ai tout l'amour. 
Mère des doux plaisirs, favorable Déesse,

Toi que suivent toujours les feux \& la tendresse, Je t'implore aujourd'hui. Si d'une tendre voix J'ai quelquefois chanté la douceur de tes loix, Si j'ai vanté ton fils, ses traits \& son empire, Et porté dans le cœur les flâmes qu'il inspire,

[suite de la note :] exemple un endroit peu digne de sa plûme dans une de ses lettres en vers adressées au Roi de Prusse. C'est lorsqu'il invite le Monarque à s'exposer moins aux dangers de la guerre.

« Songés que les boulets de vous respectent guères,

Et qu'un plomb dans un tube entassé par des sots

Peut casser d'un seul coup la tête d'un Héros. »

Je compare ce vers avec ceux de la tendre Amante du Marquis de Pesée.

«Un homicide plomb avec impunité

Frappe sans respecter l'âge ni la beauté ».

Ces derniers ont un caractère de tendresse \& de vérité qui ne dément point le stile de la Pièce. Ceux de Monsieur de Voltaire présentent une idée triviale et burlesque. Le stile marotique ne va pas dans une Epitre en vers dont le sujet est sé-

[p. 391]

Vole, descend des cieux, sers toi de ces regards

Qui savent, quand tu veux, désarmer le Dieu Mars.

Obtient qu'à mon amant il ne soit point funeste.

Mais que dis-je insensée ! \& quel espoir me reste.

En voyant cet objet de mes voeux les plus doux,

Tu serais ma rivale, \& Mars serait jaloux,

Parmis tant de frayeurs, c'est toi seul que j'implore,

Souviens toi, chèr Amant, que mon ame t'adore,

Que tu dois de mes pleurs faire tarir le cours,

Qu'en exposant ta vie, il y va de mes jours.

[suite de la note]rieux, surtout lorsque cette Epitre est adressée à un souverain. Le même Auteur avait dit plus haut pour faire l'éloge du Roi.

« Tour-à-tour la terreur de l'Autriche \& des sots,

Fertile en grands projets, aussi bien qu'en bons mots. »

Associer l'Autriche \& les sots dans un même vers, me semble une impertinence ; d'ailleurs c'est un piètre éloge pour quelqu'un de dire qu'il fait peur aux sots, c'est presque annoncer sa faiblesse puisqu'on est sot de le craindre. Quant au vers suivant, les grands projets, \& les bons mots vont mal de compagnie, ils ne sont point Analogues l'un à l'autre, la première partie du vers peint un grand-homme, la seconde un mauvais plaisant. Serait ce une faute d'impression, que cette faute de bon sens ? Je suis tenté de le croire. 
[13 juillet 1755, t. $3, \mathrm{n}^{\circ} 28$, p. 649-655]

\section{SUITE DE L'HISTOIRE DE BIENFAISANTE REINE DES FÉES.}

Je me me serais jamais imaginé, repartit Bienfaisante, que le talent de l'auteur de fût pas suffisant pour faire vivre celui qui le possède; ne vous procure-t'il pas des ressources gracieuses, des conaissances utiles ? Il y a auteurs \& auteurs, répondit cet homme. Je n'ai pas assés de talens pour produire ces ouvrages brillans qui nourrissent leur maître ; un qu'en dira-t'on, par exemple, un Siècle de Louïs XIV, un Petit-Maître de Philosophe, un Cosmopolite, une Margot la Ravodeuse, un Opera, une Tragédie. Je ne puis tirer de ma cervelle que de l'utile, du raisonable du simple, \& cela ne donne pas de l'eau à boire. Vous me surpenés de plus en plus lui dit Bienfaisante \& de quoi traittent dont ces brillants ouvrages dont vous m'avés nommé les tîtres ? comment morbleu, repliqua l'auteur, de quel pays venés-vous donc, pour [p. 65o] ignorer le contenu de ces livres. Ce sont des bagatelles obscènes dites avec légèreté, de vieilles pensées habillées d'un langage neuf \& apropriées à celui qui les débite comme à l'héritier de tout le monde; des sentimens erronés parés du nom de philosophie ; un tissu d'impostures hardies, présentées comme une véritable histoire, des anecdotes hazardées, contredites par les annales \& les fastes du tems; des fables en chanson, des déclarations amoureuses, des déclamations de vertu, des reconaissances, des... que sais-je moi : mais les éditions disparaissent et se multiplient, surtout chés l'étranger ; car le pays où vit un auteur n'est-jamais celui ou ses ouvrages fleurissent. Je m'avisai l'an passé d'en donner un de morale; mais de ces morales enjouées, propres à nourrir le cœur en récréant l'esprit ; l'impression me coûta 8o Pistoles c'était pour mon bien ; encore en devais-je la meilleure partie à un Patron : \& bien, le libraire en a vendu partout à son bénéfice, peut-être mille volumes, tandis que deux-cent-cinquante, que j’ai tiré pour mon compte, moisissent encore actuellement au fond d'une valise ; si j'en excepte une vingtaine d'exemplaires dont je dois encore la reliure, que [p. 651] je distribuai à quelques-uns de mes amis qui s'étaient chargés de prôner l'ouvrage ; mais ce fut en pure perte. Ne pouriés-vous pas, reprit la Fée, qui mourait d'envie de rire, trouver quelque libraire charitable \& honête-homme qui voulut se charger de vos ouvrages? vous venés, sans doute de Monomotepa, ma bonne mère, répondit l'auteur, en demi en colère. Ou bien il vous plaît d'ignorer ce qui est connu de tout le genre humain. Rien n'est si dur ni si arabe qu'un imprimeur vis-à-vis d'un auteur indigent. Si vous pouviés imaginer à quelles basseses un auteur est quelquefois contraint pour obtenir seulement le triste emploi de correcteur, vous vous étoneriés qu'un galant-homme puisse suporter de telles extrémités ; aussi ne suis-je plus qu'un squelette, \& si le ciel ne met bientôt fin à mes chagrins, il faudra que j’y succombe. Mais enfin, poursuivit la Fée, ne pouriés vous pas vous procurer l'appui de quelque Grand-Seigneur à la faveur d'une Epitre Dédicatoire? oh ! pour le coup vous avés résolu de me faire devenir fou avec vos nepouriés-vous-pas? dit l'auteur, en se levant brusquement de sa place. Non, Madame, non, je ne puis rien : qui vous dit que je [p. 652] n'ai pas fait usage de ce moyen. Au surplus fachés que l'on est revenu des Épitres Dédicatoires, on ne les paie plus, \& dans 
le fond on fait fort bien ; cela multipliait les mauvais ouvrages : \& tel ne s'avisait de devenir auteur en dépit de la nature, que parcequ'il comptait sur le bénéfice de l'épitre ; il est vrai que la mienne n'eût pas l'intérêt pour objet, c'était un tribut, un homage de reconaissance que je rendais à mon Protecteur ; je vous avouerai cependant de bonne foi, que j'avais espéré, que son nom donnerait crédit à l'ouvrage, \& qu'il s'intéresserait à le faire valoir ; mais j'ai compté sans mon hôte : ainsi Madame vous voyés que tous vos expédiens font sans fruit : si vous n'avés rien de mieux à me conseiller vous ne faites qu'aigrir mes chagrins. Je n'ai pas prétendu vous fâcher, Monsieur, lui dit la Bienfaisante; je serais au contraire charmée de vous rendre service. Oserais-je vous prier de me lire quelques-unes de vos productions? mais il fait froid ici, ne pouriés vous pas me conduire chés vous? je ne vous répons pas, lui dit l'auteur, que ma chambre soit plus chaude que cette boutique, car de l'hiver il n'y a point eu de feu ; j'écris dans mon lit faute de pouvoir mieux faire ; [p. 653] mais à coup sûr vous vous échauferez en montant, car le grenier que j'occupe est au septième étage. Bienfaisante suivit l'auteur qui lui donna galament la main pour monter, \& lui offrit la seule chaise qu'il y eût dans la chambre, encore était-elle si démantibulée, qu'on ne s'y asseyait pas en sureté. Quelques ais couverts de livres \& de papiers poudreux, une table boiteuse, un pot à bierre, une bouteille qui servait de chandelier, un mauvais chalit, voilà l'inventaire exact des meubles de Monsieur BIENDISANT, detail assés semblable à celui du cîte du pauvre $C h$. de M. à la HAIE. L'auteur s'assit sur le pied du grabat \& lut quelques manuscrits à Bienfaisante ; qui ne put s'empêcher d'admirer les talens de cet Auteur disgrâcié de la fortune. Voyés-vous, Madame, lui dit-il ; au milieu de ma misère, peu s'en faut que je ne sois plus heureux qu'un Roi. Si mes ouvrages me procuraient seulement de quoi vivre comme le plus austère Anacorette, je n'en demanderais pas davantage ; j'aime le travail \& toute mon ambition se borne à m'y livrer sans distraction, rien ne me chagrine comme d'être forcé de m'interrompre au plus fort de mon ardeur pour l'étude, pour courir à des devoirs de convenance \& jouër un [p. 654] personage utile dans des lieux où je suis trop France de caractère \& trop uni dans mes façons pour pouvoir y briller.

Pendant que BIENDISANT soulageait sa douleur par ses plaintes, Bienfaisante se livrait à ses réflexions. Est-il possible, disait-elle qu'au, centre d'une ville où règne le bon goût, un homme d'un mérite solide soit oublié, \& que l'on y voie caresser un piedplat qui n'a que de l'orgueil \& du babil pour talent ! c'est pour soulager le mérite indigent que le ciel m'a fait dispensatrice de ses dons ; hâtons-nous de les répandre. Que dorénavant BIENDISANT obtienne la justice qui lui est due, que les Epitres Dédicatoires aient un meilleur succès, \& que les curieux en foule enlèvent \& s'arachent ses ouvrages ; au surplus mettons-le, quant à présent, dans une position moins étroite. Les Fées ne font jamais de souhaits inutiles ; à peine Bienfaisante avait-elle achevé le sien que le Taudis de BIENDISANT prit une autre face ; Les misérables meubles dont j'ai parlé, firent place à un ameublement simple mais commande, [p. 655] Une Bibliothèque bien garnie attira surtout l'attention du BIENDISANT, auquel le Fée adressa ces paroles : livrès-vous, sans contrainte à votre goût pour l'étude ; toutes les fois que vous prendrés le petit coffre qui est au côté droit de la Bibliothèque vous y 
Annexe: « Voltaire dans la presse francophone »

trouverés la somme que vous aurés souhaitée. BIENDISANT voulut se jetter aux pieds de la bienfaitrice, mais elle était déjà disparue.

La Suite l'ordinaire prochain.

[12 octobre 1755, t. $4, \mathrm{n}^{\circ} 40$, p. $\left.917-922\right]$

\section{L'ORPHELIN DE LA CHINE. TRAGÉDIE.}

Cette piéce vient d'être donnée par Monsieur DE VOLTAIRE aux Comédiens Français qui en ont fait la première représentation le mercredy vingt Août 1755. Le fond du sujet sur lequel le Poëte s'est éxercé est vrai, quant à l'histoire, il est tire de celle de la Chine, on peut en juger par la Tragédie du petit Orphelin que le Père DU HALDE Jesuite avait déjà donnée d'après la traduction du Père DE PRÉMARE : ce Drame chinois est à la vérité d'un gout bien différent de tous ceux des autres païs. Cette pièce est entremélée [p. 918] de chants dans tous les endroits où il s'agit d'exprimer quelque grand mouvement de l'âme. La régle des trois unités n'y est point observée, c'est une Histoire mise en Dialogues dont les différentes parties sont autant de Scênes détachées, qui n'ont d'autre liaison entre elles que celle qu'ont des actions particulières, exposées par la suite de l'histoire. Il s'âgit dans cette Tragédie informe des avantures d'un enfant depuis sa naissance jusqu'à ce qu'il ait vangé ses parens. Ainsi l'action de la piéce dure environ vingt ans.

C'est sur ce Thême défectueux que Monsieur DE VOLTAIRE a composé sa Tragédie, en l'adaptant à la Scêne Française.

Un Prince Chinois détroné par le Can des Tartares, perit avec toute sa famille, à l'exception d'un enfant de six mois qu'un de ses ministres a soustrait au massacre \& qu'il eleve sous un nom inconnu. L'usurpateur découvre que cet enfant n'a pas peri \& qu'il est dans la maison de l'ancien ministre du Prince [p. 919] qu'il à détroné. Ce Ministre avec toute sa famille étant par cet événement devenu l'esclave du Tiran, il n'hésite pas à lui redemander cet enfant derobé à ses cruelles poursuites. Le Ministre embarassé ne sait longtems quel parti prendre, mais enfin la voix sacrée du devoir, faisant taire celle de la nature, il se résout pour sauver son Prince legitime à sacrifier son propre fils. L'epouse du Ministre aprenant la supercherie \& que son fils est entre les mains du Tiran se livre à la plus amére douleur, la tendresse d'une mere étouffe le devoir, elle craint pour les jours de ce fils chèri \& se flatte de le sauver en déclarant au Tiran, le stratagême dont son mari s'est servi pour le tromper. Elle va trouver l'usurpateur, ses charmes l'etonnent, il la regarde avec attention, \& se rapellant un souvenir trop tendre il la reconaît pour l'avoir aimé chèrement autrefois. Sa passion prend de nouvelles forces,il lui reproche de lui avoir preferé son rival, \& ne consent enfin à lui rendre son fils \& à sauver le Prince [p. 920] qu'a condition que renouant ses chaînes elle acceptera sa foi \& sa main : qu'a l'égard de son mari il saura s'en défaire ou l'éloigner. Si non dit il ignorant lequel de ces deux enfans est mon ennemi, pour ne pas m'y tromper j'inmole l'un \& l'autre. Cette femme inébranlable dans la foi conjugale se reproche d'avoir livré son Prince légitime, le Tiran choqué de sa résistance entre en fureur \& n'en sort que pour paraître l'amant le plus tendre et le plus soumis. Dans cette perplèxité cette femme voïant que le jeune Prince, son mari, \& son fils ne peuvent éviter l'échafaut, demande pour grace de pouvoir parler à son mari avant de prendre 
une derniére résolution. Elle obtient sa demande \& dans son entretien avec son mari elle lui déclaire l'ambarras ou elle se trouve réduite par son imprudente tendresse de Mère, elle ajoute qu'elle est incapable de manquer à son devoir, qu'il seroit lâche d'attendre la mort, \& plus glorieux de se la donner l'un à l'autre, mais craignant que la faiblesse de son sêxe ne lui [p. 921] fasse porter des coups mal assurés : J'attens dit elle de vous ce service, \& vous crois assés généreux pour me suivre : son mari lui représente qu'enchainé \& sans armes il est dans l'impuissance de seconder ses vœux, elle lui présente un poignand en disant frappe voilà mon sein, le Mari leve un bras homicide que l'amour arrête, elle l'encourage, il le leve une seconde fois, l'usurpateur entre \& le retient, il reproche au Mari sa cruauté, dit à la famme que sa vertu triomphe \& le confond; qu'il veut que tous deux vivent libres, heureux \& ses amis, se déclarant le Protecteur du Prince \& le Père de leur fils.

Le petit Prince n'y le fils du Ministre ne paraissent pas dans la piéce. - On dit que les trois premièrs actes sont admirables, les deux derniers plus faibles. On trouve la conversion du Tiron trop précipitée. Il y à dans le courant de la piéce des sentences de vertu \& de réligion parfaitement bien traitées, on croit qu'elle fera [p. 922] charmante la lécture. Comme les Comédiens Français répugnaient aux frais des habits Monsieur DE VOLTAIRE leur a laissé sa part d'auteur pour y fournir : tant il est vrai qu'on ne trouve pas toujours un chef de troupe assés désinterèssé pour faire valoir le Théatre aux dépens de sa bourse \& que l'on doit faire un cas particulier de ceux qui népargnent rien pour l'eclat \& la décence du spectacle. On ignore encore si cette piéce est un fruit des réveries de la petite maison d'Epicure $\mathcal{E}$ du jardin d'Aristippe, mais les sentimens qui y règnent l'annoncent $\&$ tiennent un peu l'hermite.

[14 décembre 1755, t. 4, n49, p. 1117-1131]

\section{COMMENTAIRES SUR LA TRAGEDIE DE L'ORPHELIN DE LA CHINE}

Par Mr. de VOLTAIRE

Ce titre annonce une certaine Majesté tragique qui ne paroît pas aux faibles regards du commun des grands esprits. L'on s'imagineroit peut-être au premier point de vue qu'il ne doit interresser que ces bonnes grandes meres dont tous les organes usés ne peuvent être mûs que par des objets dignes de pitié. Mais comme [p. 1118] dit fort bien le grand Blaise, la Pitié est l'Heroïsme accompli, \& nous ajoutons aprez Augustin le Chimerantique, que la Pitié seule dans le monde est le Nerf de toutes les grandes actions comme cet actiome semble le prouver. Une grande ame ne se montre jamais mieux \& d'une façon plus brillante que lorsqu'elle peut faire des actes éclatans de misericorde \& de pitié. C'est aussi delà que tous les grands Guerriers n'ont jamais rien plus à cœurque de faire des malheureux, pour faire éclater davantage la bonté de leur cœur en pardonnant aux Vaincus, en faisant quelque gratifications à ceux qu'ils ont ruinés en prenant soin des Orphelins, lorsqu'ils ont fait massacré les peres \& meres. Qui n'admirera un si grand fond de clemence? Suivant la judicieuse reflection de Barbara Clementin, dans son livre de la cruauté salutaire. L'auteur de notre Orphelin ou plutôt son espece de pere putatif, car il avoue que d'autres, l'ont engendré avant lui, est admirablement [p. 1119] bien entré dans son divin traité Contraria contrariis curantur. Il croit avec lui qu'il est de toute necessité d'être mechant pour devenir bon : 
qu'il faut être ignorant pour devenir sçavant : qu'il faut être malheureux pour devenir heureux : qu'il faut, que dire plus! Etre Batard ou Orphelin pour avoir le bonheur de voir les autres hommes s'etonner qu'un tiran meurtrier de l'Empereur son père se fasse elever comme un fils de bonne famille à l'ombre du thrône qu'il a usurpé sur lui. Tel est le sublime interêt, tels sont les magnanimes sentimens \& le merveilleux denouëment que l'Auteur fait habilement resulter d'un titre aussi peu parlant que celui d'Orphelin de la Chine. C'est comme si l'on disoit le Batard de la France. Quelle vaste \& indeterminée pensée ne donne-t-on pas !

Publicus le Censeur étourdi par ces explications eclairés \& lumineuses, veut étançon-[p. 112o]ner sa critique en soutenant qu'excepté trois ou quatre Auteurs ; qui à force de vouloir viser dans le singulier sont tombés dans l'extravagance, on a toujours intitulé les pieces du nom des Heros qui y joüent les principaux personages : Que la scene peut bien soufrir deux ou trois heros à la fois comme les deux freres ennemis de Racine, Alexandre \& Porus \&c. Mais que l'on n'a jamais oui dire qu'une piece de theâtre put porter le nom d'une personne quî n'y paroît pas plus que le petit Orphelin de Chine.

Superbus le fantastique dresse alors les oreilles \& repond en baillant que cela se peut pourvû que les Acteurs parlent de la personne d'une manière qui excite la pitié. Il conclut même qu'un aussi celebre genie que Mr. de Voltaire est en droit de se tracer vers la gloire des routes tout a fait escarpées \& qui lui doivent être particulierement réservées. Qui doute ajoute Blaise le Grand, dans son 6 [p. 1121] livre des cas reservés, qui doute que ce Heros des la Littérature est superieur aux regles du theatre, à ces sottes regles qui prescrivent dans une piece l'unité de lieu \& d'action dans tout au plus 24 heures. Qui n'entrevoit même qu'en nouveau créateur il pourra faire naitre un jour quelqu'enfant qui au bout des 24 heures aura fait l'amour, combattu ses ennemis, \& cessé de vivre aprez cent ans d'un heureux \& magnifique Regne.

Se peut-il qu'en ce temps de desolation

En ce jour de carnage et de destruction

$\mathcal{E}$ c. $\mathcal{E}$.

Que ces premiers vers d'une Tragedie, où il s'agit sublimement d'un petit Orphelin, sont bîen propres à faire pitié ! Qu'ils debutent habilement dans une piece où tout doit être pitoyable! s'ecrie le Docteur Barbarus Clementin. Arïstotes \& Platon, apres eux Horace \& Boileau dans leur Art Poetique ont enseigné à ceux qui ne le sçavoient pas, qu'un Ecri-[p. 1122] vain doit toujours ajuster son stile au sujet badin; le grave au serieux; le galant à l'amour, le magnifique à l'heroique; le puerile à l'enfantin ; les termes de desolation $\mathcal{E}$ de destruction; un stile enfin pedammant gemissant quand il s'agit de quelque cruelle catastrophe. Admirons donc Monsieur de Voltaire, s'ecrie Innocentius stupefait avec tout l'anthosiasme que Madame Dacier fait voir pour les belles sottises qui brillent de tems en tems dans son Homere de la France cet exacte Ecrivain qui a si bien fait prendre à son stile les couleurs de son Orphelin qu'il s'y montre tout a fait pitoyable. Publicus le Censeur objecte cependant qu'on peut craindre aussi que ses trop grans admirateurs ne deviennent aussi trop pitoyables, qu'à force de vouloir trouver trop de patetique dans l'orphelin ils n'en viennent à se faire pitié à eux même en se chagrinant trop pour le petit heros enfant, moins infortuné qu'eux, puisqu'on n'est malheureux qu'à proportion du [p. 1123] sentiment de son malheur qui n'est rien si on ne le connoit pas. Le Grand Blaise qui est le Paranimphe de tous les sentimens qui tendent à la pitié repond que cette critique auroit tombé d'elle- 
même à la vüe du Heros emmailloté ; si l'on avoit seulement eu la consolation de le voir vivant sur la scene aprez avoir si fort tremblé pour sa mort. Ils meritoient bien du moins qu'on leur montrat par curiosité ce petit Heros à la fin de la piece.

Une premiere \& longue scene nous apprend que les Tartares qui riment avec Barbares ont subjugué les Chinois Troupeau faible $\mathcal{E}$ timide par un guerre homicide. Publicola Bonsens demande avec étonnement, si ce mot d'Homicide n'est pas une cheville, \& s'il y a des guerres où l'on ne tuë point des hommes, s'il y a en un mot des guerres qui ne soient point homicides. Il croit bien qu'on peut leur donner l'epitéte de sanglantes, cruelles ou [p. 1124] legeres, acharnées ou passageres pour caracteriser la juste valeur des coups des soldats, l'activité ou la lenteur des operations du General. Mais il ne peut concevoir qu'il y ait des guerres où l'on ne tue personne. Blaise le Grand qui est toujours porté à fuir piteusement les objets cruels, dit que Voltaire traitant l'histoire d'un orphelin a eu peur qu'on ne prit la guerre dont il parle pour une guerre d'enfant où il y a tout au plus quelques bosses à la tête \& dix ou vingt machoires disloquées. C'est pourquoi l'Auteur dit-il, a trez habilement employé son terme de guerre homicide pour faire voir que ce n'etoit pas un jeu d'enfants. Mais Superbus le Phantastique reprend aigrement ce grand Blaise \& prend tout un autre tour pour expliquer ou deviner la sublime idée de l'Auteur dans son expression de Guerre Hommicide. Il dit avec son hardiesse accoutumée que l'on a vû chez les peuples les plus belliqueux des guerres momentanées sans effusion de sang. [p. 1125] Publicus le Censeur pousse sur cela Superbus le Phantastique \& il lui objecte que les guerres inhomicides ne sont chez les hommes raisonables que des levées de bouclier, Mais que par l'ordre des Souverains ces boucliers retombent avant que les bras soient fatigués de les porter, \& avant que la main ait touché la poignée de l'épée. Car il est toujours mieux de ne rien précipiter quand il s'agit de mourir. Tout homme de bon sens le croit ainsi. Superbus le Phantastique soutient au contraire qu'on peut faire la guerre en imagination \& comme les Gazettiers affectés au parti de quelque Puissance Belligerante tuer autant d'hommes qu'il leur est desirable.

Publicola Bonsens vient à la traverse de deux critiques commentateurs \& met entre eux deux une barriere qu'il croit inforçable, ce mot n'est pas trop Français. Qu'importe l'usage le rendra aussi galant que celui d'inexpugnable. La mode des habits \& des termes [p. 1126] est une nouveauté qui etablit l'usage jusqu'à nouvel ordre.

Vesputius Vestitus voix de fer dans son traité de Casibus dixmillieme commentaire ( ${ }^{*}$ 'on compte jusqu'à neuf mille commentateurs sur Aristotes) des Misteres d'Aristotes excite le critique Publicola Bonsens, à dire naturellement qu'il connoît une grande Souveraine qui par son courage, par sa haute sagesse \& par son attendrissante clemence a repris dans une nuit le throne d'un vrai Hero son Père, sans que Sa main sacrée ait rougi Son sceptre d'une seule goutte de sang, dans une revolution aussi subtile qu'elle étoit désirable. Mais aussi avoue t-il que cet exemple est un trait unique dans l'histoire. Carolus Manutenens Villam admire cette immortelle Heroine \& à feuileté toute l'histoire pour lui trouver son paralelle, \& cent mille \& mille volumes les Romans mêmes selon lui n'ont point de si-[p. 1127]tuations aussi hardies \& aussi charmantes que les vèrités qui brillent dans l'histoire de la FILLE de PIERRE LE GRAND. Publicola Bonsens \& Publicus le Censeur soutiennent donc qu'une aussi unique et merveilleuse exception a la regle n'empêchent point de faire triompher par tout ailleurs la critique d'une guerre homicide. 
Annexe: «Voltaire dans la presse francophone »

Mais laissons triompher Publicola Bonsens de l'Auteur, \& n'en admirons pas moins la rime énergique d'une Guerre Homicide. Avançons \& nous trouverons de plus vastes chams pour couronner de fleurs notre energique Auteur.

Avec quelle dexterité Monsieur de Voltaire n'amene t-il pas l'histoire interressante de l'amour du conquerant Gengis-Kan pour la belle Idamé \& qu'il avoit aimé lorsqu'il n'etoit encore qu'un pauvre Scyte vagabond \& refugié pour lors dans la Chine. Cette femme devenuë le partage du vertueux Zamti nous [p. 1128] fait elle-même le recit des refus humilians \& des mepris qu'il eut à soufrir. Severus Pedant semble regarder avec un front austere les magnifiques louanges que dans cette piece la vertueuse Idamé donne à ce barbare qu'elle recoit pour sont [sic !] amant. Augustin le Chimerantique passe au contraire à la vertueuse epouse du respectable Mandarin Zamri cet Amour dont son coeur en secret sapplaudit parce qu'elle a l'attention d'en faire un mistere à son mari. Cette decence tres ordinaire aux femmes de bon sens, le racommode avec Idamé. Il lui pardonne aussi la vanité qu'elle tiroit de la passion du Scyte en faveur de l'intention qu'elle avoit d'en faire un bon soldat; de le convertir à la religion Chinoise. Religion, dit fort exactement l'Auteur, Religion de tout tems épurée. Qui doute aprez cela qu'une religion où l'on adore les Idoles avec autant d'ardeur que de scrupule dans les ceremonies, n'authorise Idamé, quoi qu'epouse d'un autre à s'applaudir encore d'un [p. 1129] amour qui auroit pu gagner le paradis à un pauvre Scite fugitif s'il avoit voulu changer de religion \& adorer les Idoles Chinois. C'est ains, ajoute Severus Pedant, que les Docteur d'emor \& entr'autres les Moines Seitusei ont raison de soutenir que pour la propagation de la croyance Emoraine \& pour le bien des ames on doit tout employer jusqu'au mensonge, jusqu'au fer \& au feu.

Ces moyens que quelque fou Philosophe croiroit un peu violens en depit de ce mot coge illos intrare forcez les d'entrer ; ce fer \& ce feu dis-je n'ont pas toujours été mis en usage par les plus grands faints ; \& plusieurs ont été galamment s'exposer aux douceurs de l'amour pour convertir des pauvres filles prostituées dans des lieux publics \& dont la beauté avoit facilité le libertinage. Qui revoquera, s'ecrie alors Augustin le Chimerantique que la vertu de la belle epouse de Zamti ne jette les plus beaux feux lorsqu'elle paroît [p. 1130] souhaiter d'avoir étouffé dans ses bras le courage du Beau \& Vaillant Temugin, courage si fatal aux Chinois. Qui doute qu'elle ne fasse une action meritoire en insinuant qu'il ne tient à rien qu'elle ne cherche à retenir dans son sein ce Temugin devenu le conquerrant Gengis-Kan \& cela pour l'empêcher de poursuivre plus loin une cruelle victoire sur ses compatriotes desolés \& sur une religion épurée pour la quelle l'on n'a rien de trop précieux à sacrifier.

Publicus le Censeur \& Puplicola Bonsens toujours opposés à Augustin le Chimerantique pretendent qu'il y a de l'absurdité à vouloir faire de l'amour une espece de predicateur sur la morale \& sur la Religion. L'amour n'etant qu'un sentiment qui rapporte tout à un seul point de vuë \& qui loin d'aimer à reprendre \& convertir admire comme des vertus les vices de son objet \& qui en nouveau Salomon encense les Idoles de ses Maitresses. [p. 1131] En un mot ils soutiennent qu'il faut distinguer l'amour de l'amitié ; L'amitié trouvant à reprendre jusques dans les vertus, \& l'oeil de l'amant ne voyant que les vices que comme des qualités admirables. 
Le reste paroitra successivement dans les feuilles suivantes.

\title{
II. Voltaire dans le Journal des sciences et des arts, Moscou, $1762^{2}$
}

[Exlibris Bibliothèque de Voltaire arm 8 № 292. Reliure demi cuire, cuire brun, papier brun pointé par noir].

[p. 1]

Journal des sciences et des arts. No. 1.

\author{
C'est avec confiance qu'on commencera ce second \\ Volume par un Extrait qui interesse tous les \\ Nationaux \& tous les membres de la \\ Republique des Lettres. \\ Memoires sur l'Empire de Russie par M. de Voltaire \\ Premier Volume.
}

On ne s'attend pas sans doute que je fasse un Extrait suivi de cet ouvrage ; les grandes actions de Pierre Premier ont été si immortalisées, elles sont si gravées dans le coeur de ses fideles sujets, le Gouvernement de son Auguste Fille nous les retrace si sensiblement, que ce seroit un soin superflu. Il ne doit donc etre question que de la maniere dont M. de Voltaire a traitté ce sujet. On ne risque rien en promettant qu'on reconnoitra toujours le grand homme dans ce nouvel ouvrage. On y retrouvera ces traits frappans, ce jugement sûr, cet Esprit Philosophique qui a toujours percé dans ce qu'il a fait.

Constamment ennemi du faux, M. de Voltaire continue dans sa Preface a nous prevenir contre tous ceux qui en ecrivant l'histoire, ont sacrifié la verité a l'esprit de parti, a l'adulation, ou a des vuës mercenaires. Il est bon de faire connoitre ces auteurs qu'il proscrit. Ce sera un preservatif sûr pour ceux qui entrent dans la carriere historique; l'histoire de Pierre Premier par le B. Nestesuranoy, les memoires de Colmenar sur l'Espagne, l'histoire de l'Empereur Charles 6. voila quelques uns des [p. 2] livres que nous voions a l'Index redoubtable du Heros de la litterature. En recompense, il donne pour modeles, l'histoire de M. de Thou \& celle de Rapin Toiras.

Jaloux d'exactitude en ce genre, M. de Voltaire rapporte un Certificat que sa Majesté le Roi Stanislas lui a donné du quel il resulte que sa Majesté n'a rien trouvé que de conforme a la verité dans l'histoire de Charles Douze.

Ce n'est pas sur des fondemens moins certaines qu'ont été ecrits les Memoires dont on fait ici l'extrait ; M. de Voltaire nous apprend comment Son Excellence M. Iwan Iwanowitz de Schouvalow l'a mis en etat de travailler a l'histoire de Russie. "Enfin un Chambellan de L'IMPERATRICE ELISABETH FILLE DE PIERRE, a été l'instituteur d'une Université depuis quelques années. C'est le même qui m’a fourni tous les memoires sur lesquels j'ecris ; il etoit bien plus capable que moi de composer cette histoire, même

\footnotetext{
${ }^{2}$ Ce texte a été découvert dans la bibliothèque de Voltaire, qui est conservée à la Bibliothèque nationale de Russie, par Vladimir Somov qui l'a saisi. J'en profite pour le remercier pour la permission de publier ce texte ici. Je remercie également les collaborateurs de la Bibliothèque Voltaire et notamment sa directrice Natalia Speranskaïa pour leur accueil et leur disponibilité.
} 
Annexe: «Voltaire dans la presse francophone »

dans ma langue. Tout ce qu'il m'a ecrit, \& que j’ai deposé dans la Bibliotheque publique de Geneve fait foi que ce n'est que par modestie qu'il m'a laissé le soin de cet ouvrage.

Avant d'en venir au Regne de Pierre Premier, on trouve une Description de la Russie; je recueillerai ici les faits, Epoques \& particularités qui m'ont paru meriter l'attention des gens de lettres.

«C'est en 1689. que le Premier Traitté avec la Chine a été conclu. Il a été écrit en latin. "Voila encore un hommage glorieux \& moderne qu'on a rendu a cette langue ».

«Si l'on voiageoit en droite ligne depuis Petersbourg par Dantzic, Hambourg, Amsterdam, jusqu'aux extremités Septentrionales de la France, on ne trouveroit pas un montagne.

«L'usage de l'ecriture n'a eu lieu en Russie que depuis le 5. Siecle.

« A la fin du dernier Siecle, l'on ne calculoit encore en Russie qu'avec des boules. C'est un Mathematicien anglois nommé Ferguson qui a introduit sous Pierre I. le calcul.

[p. 3] On voioit des Indiens sur les confins de l'Allemagne ; ce Phenomene paroitroit incroiable, si M. de Voltaire n'en fournissoit pas une explication très plausible. «Ces Indiens s'embarquoient sur la Mer d'Hircanie, remontoient le Volga, navigeoient [sic] sur le Kama, traversoient les Terres jusqu'a la grande Permie, \& s'embarquoient sur la Baltique. Voila un arrangement auquel n'avoient point pensé ceux qui croient cette peregrination impossible.

«On Compte dans l'Empire 740o. moines \& 560o. Religieuses.

La Siberie a été decouverte en 1563.

«C'est en 1698 que l'ordre de S. André a été institué. Le commencement de l'année a été fixé au Premier Janvier, l’an 1700.

Les Jesuites ont été exclus de la Russie par un Edit de 1718.

Le tiers du Regiment de le Fort de 12000. hommes etoit composé de François Refugiés.

«Il y a plus de 500. mille habitans a Moskou \& 24. millions d'habitans dans la Russie. On trouve a cette occasion une liste de tous ceux qui paient la contribution. Ils sont au nombre 664000o. \& l'on ne comprend point dans ce nombre les femmes, les filles, les Enfans, l'Etat militaire, la Noblesse ni les Etrangers.

Entre les faits Anecdotes que M. de Voltaire rapporte, on n'oubliera pas celui ci qui etablit si bien la magnanimité de notre Heros. Lorsque Charles 12. forma, après la Bataille de Pultava, le projet de se retirer chès les Turcs, Pierre lui ecrivit pour lui offrir la paix, lui promettant même de ne pas le retenir prisonnier. C'etoit precisement le moment ou il n'etoit nullement besoin de menager le Roi de Suede. Au reste il etoit deja parti, lorsque l'exprès qui lui portoit cette lettre arriva.

Veut on voir quelque portrait? je transcrirai celui de M. le Fort, nom qui doit être si cher a la Nation.

[p. 4] « Pierre donna sa confiance a un Etranger. C'est ce celebre Le Fort d'une noble $\&$ ancienne famille de Piemont transplantée depuis près de 2. Siècles a Geneve, ou elle a occuppé les premiers emplois. On voulut l'elever dans le negoce, qui seul a rendu considerable cette ville, autrefois connue uniquement par la controverse.

«Son genie qui le portoit a de plus grandes choses lui fit quitter la maison paternelle dès l'age de 14. ans. Il servit quatre mois en qualité de cadet dans la citadelle de Marseille. De là, il passa en Hollande, servit quelque tems Volontaire, \& fut blessé au Siege de Grave. Cherchant ensuite son avancement partout ou l'esperance le guidoit, il s'embarqua en 1675. avec un Colonel Allemand nommé Verstein, qui levoit quelques 
soldats pour Alexis dans les Pays Bas. Quand ils furent a Archangel, Alexis n'etoit plus. Le Gouverneur d'Archangel laissa longtemps Vesrtein, Le Fort \& sa troupe dans la plus grande misere \& les menaça de les envoier au fond de la Siberie. Chacun se sauva comme il put.

«Le Fort, manquant de tout, alla a Moscou \& se presenta au Resident de Dannemark, nommé de Horn, qui le fit son Secretaire. Il y apprit la langue Russe, fut presenté au Czar Pierre qui lui donna d'abord une Compagnie d'Infanterie. Apeine avoit il servi. Il n'etoit pas savant ; il n'avoit etudié aucun art, mais il avait beaucoup vu avec le talent de bien voir ; sa conformité avec le Czar etoit de devoir tout a son genie. Il s'attacha a Pierre, les plaisirs commencerent la faveur \& les talens la confirmerent.

Après avoir rendu compte de la partie historique, venons a celle ou l'on reconnoitra encore mieux l'Auteur de l'Essai sur l'histoire Universelle. Ce sont ces phrases expressives, ces images fortes, ces maximes, ou souvent d'un seul mot, il censure un vice, il attaque un ridicule, il corrige les hommes \& les porte au bien \& a la verité avec une force irresistible. Je ne me suis pas permis de les rassembler ici toutes, y en aiant quelques unes d'une nour[p. 5] riture un peu trop forte, mais on ne trouvera qu'a approuver dans celles qui suivent.

« Ne dites a la Posterité que ce qui est digne de la Posterité.

«Aucune famille sur la terre ne connôit [sic] son premier auteur, \& aucun Peuple ne peut savoir son origine.

« Tout Peuple qui n’a point cultivé les arts doit etre condamné a etre inconnu.

«On juge aujourd'hui que Charles Douze meritoit d'etre le Premier soldat de Pierre le Grand ; l'un n'a laissé que des ruines. L'autre est un fondateur en tout genre.

L'ancienne coutume etoit de ne se presenter ni dans l'eglise, ni devant le thrône avec une epée, coutume orientale opposée a notre usage ridicule \& barbare d'aller parler a Dieu, aux Rois, a ses amis \& aux femmes avec une longue arme offensive qui descend au bas des jambes.

A l'occasion d'un chef de Cosaques pendu : "Cette partie du monde etoit celle ou les hommes etant le moins gouvernés par les mœurs ne l'etoient que par les supplices \& de ces supplices affreux naissoit la servitude \& la fureur secrette de la vengeance.

Parlant des Siecles precedens. On ne savoit point alors deguiser l'orgueil par les dehors de la bienseance.

Sur la Conjuration des Strelitz. "Les Troupes passerent enfin de la fureur a la crainte \& de la crainte a la plus aveugle soumission, changement ordinaire a la multitude.

Une Phrase fait son Parallelle entre les Princes Ivan \& Pierre Premier. « Ivan n'avoit que le nom de Czar, \& Pierre agé de 17. ans avoit deja le courage de l'etre.

Sur l'introduction de l'Arithmetique. «L'Empire de la Russie ne la reçut que mille ans après les autres Européens. C'est le sort de tous les arts ; ils ont fait lentement le tour du monde.

[p. 6] « On observoit en Russie \& l'on y calculoit les Eclipses, tandis que dans la patrie de Galilée, des ignorans ordonnoient a des ignorans de croire la terre immobile.

«On ne desarme ses Ennemis que par la force. On les enhardit par la foiblesse.

Quand il parle d'un hopital bati par Pierre I. " vaste hopital, non de ceux qui encouragent la faineantise \& qui perpetuent la misere, mais ou quiconque est renfermé devient utile. 
Annexe: «Voltaire dans la presse francophone »

En se plaignant de l'inexactutude des Geographes. « L'ambition a jusqu'ici pris plus de soin de devaster la terre que de la decrire.

«Sur la jonction des mers Baltique, Caspienne \& Noire; il y là plus de gloire veritable que dans le gain d'une bataille.

«Voici quelques traits qui peignent l'Illustre Pierre. Vainqueur des Turcs \& des Tartares, il voulut accoutumer son Peuple a la gloire, comme aux travaux.

«En faisant fouiller les mines de Siberie, il travailloit à enrichir ses Etats \& à les deffendre.

«Le charpentier de Sardam promit trente mille hommes au Roi Auguste. Cette expression fait souvenir du vers ou Sixte quint est designé par ces termes, le Pâtre de Montalte.

Mr. de Voltaire dit d'Auguste Roi de Pologne «quand on songe qu'avec cette foiblesse, Auguste etoit un des plus braves Princes de l'Europe, on voit bien que c'est le courage d'esprit qui fait perdre ou conserver les Etats, qui les eleve ou les abaisse.

Les malheurs de Charles 12. en Turquie etoient selon M. de Voltaire "plus dignes d'un Héros de l'Arioste que d'un Roi sage.

M. de Norberg historien de Charles 12. n'est point oublié. «Les Suedois crierent dabord victoire a Pultava ; Norberc

[p. 7] « qui etoit, loin du champ de bataille, au bagage ou il devoit etre, pretend que c'est une calomnie.

Mr. de Voltaire se range du parti de ceux qui pensent qu'il seroit mieux de n'infliger la peine de mort a aucun coupable, question qui a deja été traittée dans ces feuilles, p. 39. du Premier volume. C'est a l'occasion du Deserteur Jacob ; voici ses termes.

"Cet exemple fait voir que l'humanité qu'on exerce aujourd'hui en Russie est preferable aux anciennes severités \& retient mieux dans le devoir les hommes, qui, avec une education heureuse, ont pris des sentimens d'honneur. L'extrême rigueur etoit alors necessaire envers le bas peuple. Mais que les moeus ont changé ! L'Impératrice ELIZABETH a achevé par la clemence l'ouvrage que son Pere commença par les loix. Cette indulgence a même été poussée a un point dont il n'y a point d'exemple dans l'histoire d'aucun Peuple. Elle a promis que pendant son Regne, personne ne seroit puni de mort, \& a tenu sa promesse. Elle est la premiere Souveraine qui ait ainsi respecté la vie des hommes. Les malfaiteurs ont été condamnés aux mines, aux travaux publics. Leurs chatimens sont devenus utiles a l'Etat, Institution non moins sage qu'un humaine. Partout ailleurs, on ne fait que tuer un criminel avec appareil, sans avoir jamais empêché les crimes. La terreur de la mort fait moins d'impression peutêtre sur des mechans pour la plupart faineants, que la crainte d'un chatiment \& d'un travail penible qui renaissent tous les jours. 